\title{
High-performance detection of alcoholism by unfolding the amalgamated EEG spectra using the Random Forests method
}

\author{
Thilo Rieg \\ Aalen University \\ thilo.rieg@studmail.htw-aalen.de \\ Marius Hitzler \\ Aalen University \\ marius.hitzler@studmail.htw-aalen.de
}

\author{
Janek Frick \\ Aalen University \\ janek.frick@studmail.htw-aalen.de \\ Ricardo Buettner \\ Aalen University \\ ricardo.buettner@hs-aalen.de
}

\begin{abstract}
We show that by unfolding the outdated EEG standard bandwidths in a fine-grade equidistant 99-point spectrum we can precisely detect alcoholism. Using this novel pre-processing step prior to entering a random forests classifier, our method substantially outperforms all previous results with a balanced accuracy of 97.4 percent. Our machine learning work contributes to healthcare and information systems. Due to its drastic and protracted consequences, alcohol consumption is always a critical issue in our society. Consequences of alcoholism in the brain can be recorded using electroencephalography (EEG). Our work can be used to automatically detect alcoholism in EEG mass data within milliseconds. In addition, our results challenge the medically outdated EEG standard bandwidths.
\end{abstract}

\section{Keywords:}

Alcoholism, Electroencephalography, Random Forests, Spectral analysis, Machine Learning

\section{Introduction}

Alcoholism is a global addiction problem that is currently at the top of the list of addictions in many countries. The number of alcohol-dependent people in Germany is in the millions, with several tens of thousands dying every year as a result of alcohol consumption [1]. The long-term consequences of alcohol for the body, especially for the brain, are often underestimated. The comprehensive structure of the brain contains multiple systems that are responsible for the complete control of the human body. These multiple brain systems communicate through billions of neurons. The exchange of information between the neurons takes place via messenger substances, called neurotransmitters. Body reactions, feelings and mood are influenced depending on the amount and type of neurotransmitters. The brain balances the communication speed of the neurotransmitters. Alcohol, however, slows down the speed of this communication.

Regular, long-term alcohol consumption leads to changes in the neurons, such as reduction of brain cells and shrinkage of brain mass. These changes can negatively affect some abilities such as coordination, regulation of body temperature, sleep, mood, cognitive abilities and memory [2]. Electroencephalography is a widely used approach to measure the functional state of the brain. EEG generates aperiodic time series data, which indicate the registration of electrical activities of the brain. Enormous amounts of data from multichannel EEG are examined by experts for disorders and effects in the brain. However, this analysis is very time-consuming and error-prone, as no standardized criteria exist [3-4]. Therefore, there is also a need to develop and apply an automatic classification method for identifying alcoholism. At present, several methods for solving problems have been proposed, which are achieving ever better results [5]. In EEG, the spontaneous activities of the brain are particularly relevant, which can be derived by a continuous measurement of brain waves at the skull surface. The frequency range of these spontaneous activities ranges from 0.5 to $30 \mathrm{~Hz}$ and is subdivided as follows in accordance with an international convention [6]: 


\begin{tabular}{|l|l|l|}
\hline $\begin{array}{l}\text { Frequency } \\
\text { Band }\end{array}$ & $\begin{array}{l}\text { Frequency range } \\
\text { in } \mathrm{Hz}\end{array}$ & Characterization \\
\hline Delta & $0.5-3.5 \mathrm{~Hz}$ & Deep sleep \\
\hline Theta & $3.5-7.5 \mathrm{~Hz}$ & $\begin{array}{l}\text { Measured in sleep } \\
\text { and especially in } \\
\text { dream phases. In } \\
\text { brain diseases } \\
\text { such as epilepsy, } \\
\text { they also occur in } \\
\text { waking phases }\end{array}$ \\
\hline Alpha & $7.5-12.5 \mathrm{~Hz}$ & $\begin{array}{l}\text { Relaxed awake, } \\
\text { closed eyes }\end{array}$ \\
\hline Beta & $12.5-30 \mathrm{~Hz}$ & $\begin{array}{l}\text { Inner restlessness, } \\
\text { stress, } \\
\text { concentration }\end{array}$ \\
\hline Gamma & $>30 \mathrm{~Hz}$ & $\begin{array}{l}\text { Extreme } \\
\text { concentration }\end{array}$ \\
\hline
\end{tabular}

Tab. 1 Standard EEG bandwidths [7][8]

If EEG is applied to alcoholics and non-alcoholics, differences within the individual frequency bands, from delta to alpha, can be observed [9]. In the Machine Learning proposals published so far, this classification of frequency bands was taken into account and strictly followed. Mumtaz et al. [10] has already been able to make a good classification of alcoholics and non-alcoholics through linear regression on the classical frequency bands. Gopika Gopan et al. achieved good results with SVM and Fk-NN [11]. In this paper, however, the entire frequency range is considered as a whole and divided and analyzed into 99 equal sections. Due to the fine division of the frequency bands, it may be possible to identify single, highly important frequency bands that would not have emerged with the other methods.

\section{Method}

\section{Dataset and EEG noise removal}

The data set used in this paper comes from a large study investigating the correlation of genetic predisposition to alcoholism. The data set contains data from 64 electrodes on the scalp scanned at $256 \mathrm{~Hz}$. The sensor positions of the 64 electrodes are shown in Fig. 1. Altogether 122 subjects, divided into alcoholics and a control group, were examined. Each patient completed 120 tests with different stimulation. This stimulation was both visual and verbal depending on the test. In addition, the time of stimulation was also varied. The data set used was created by Henri Begleiter Neurodynamics Laboratory, State University of New York Health Center Brooklyn, New York and has no restrictions on use. Zhang et al. [12] describe in detail the data collection process. The full dataset is available from https://archive.ics.uci.edu/ml/machinelearning-databases/eeg-mld/eeg.html.
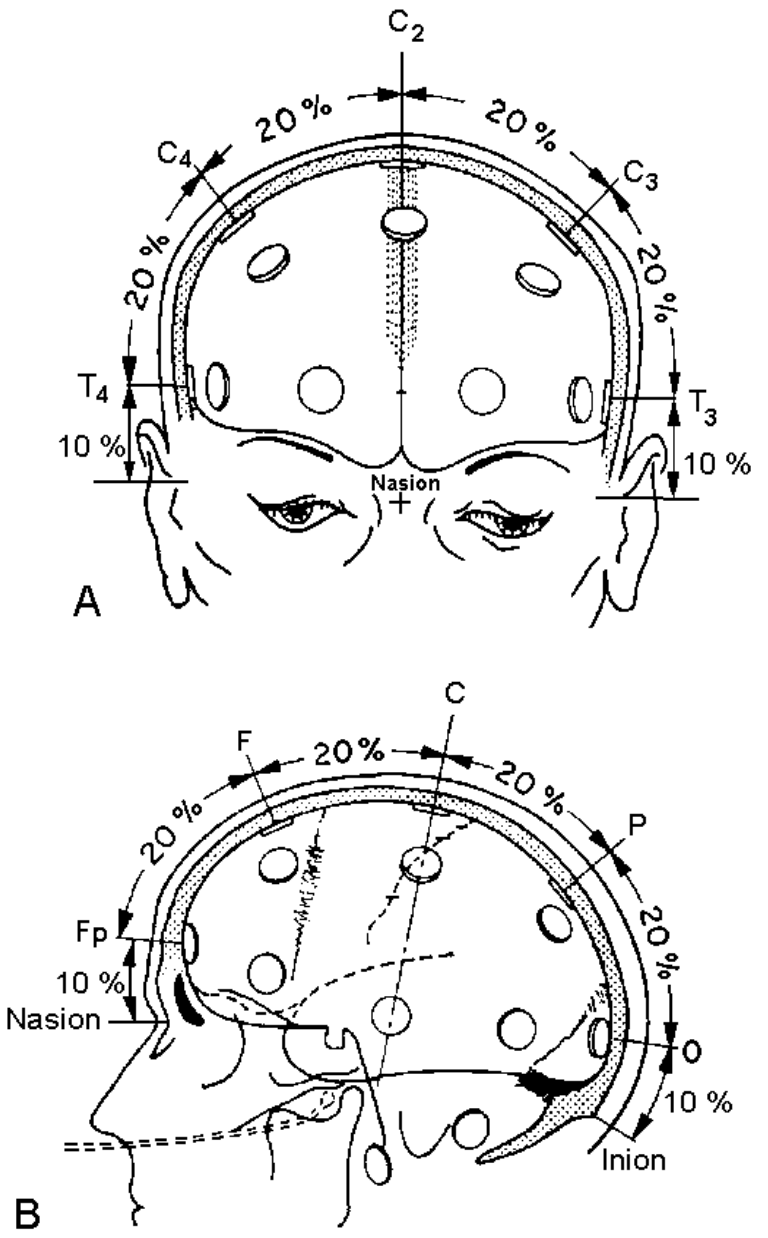

Fig. 1 EEG 10-20 system [13]

The internationally standardized $10-20$ system was used to position the electrodes. At the first international EEG congress, guidelines for the standardization of EEG measurements were proposed and implemented by Herbert $\mathrm{H}$. Jasper. This mainly relates to the exact positioning of the electrodes on the scalp [14]. The 1020 method measures the distance from Nasion to Inion on the scalp and defines it as $100 \%$. This distance is then divided in percent from the Nasion in the direction of the Inion. Starting with a $10 \%$ distance from Nasion towards the Inion, four further $20 \%$ distances follow, which then end with a $10 \%$ distance finishing at the Inion [Fig 1]. 
Fig 2 shows how the 64 electrodes are arranged according to the 10-20 system. in the EEG data [16]. Another approach was to filter out interfering signals such as blinking through Principal Component Analysis (PCA).

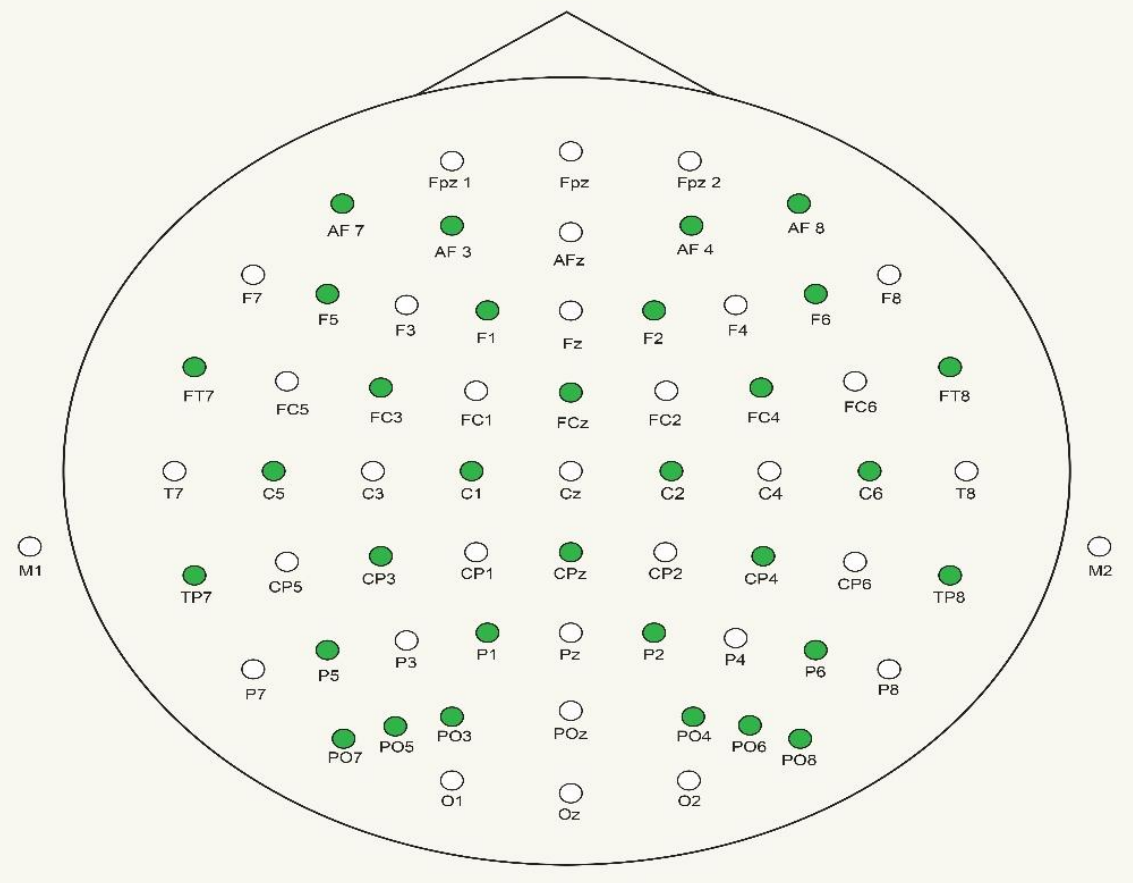

Fig. 2 EEG sensor placement on the human scalp

In order to better record brain activity, the subjects were exposed to various stimuli. Images from the Snodgrass and Vanderwart image sets have been selected for this purpose. Each subject was exposed to either a single stimulus or two stimuli. If two stimuli were shown, they were displayed in either the same or different states. Thus, the object displayed for the first stimulus could deviate from or be identical to the second stimulus. A central problem with EEG data sets is the high noise generated during data collection. The electrodes attached to the scalp must record the finest signals from the brain but which also leads to the recording of mixed signals. Above all the movement of the eyes, blinking, muscle activities as well as the heartbeat are found as disturbance data in the EEG data set [15]. The data must be cleaned up accordingly. In the history of electroencephalography, many different methods have been used for noise removal. For example, an attempt was made to remove mixed signals by regression within the time or frequency domain. However, if muscle movements are considered during EEG recording, they cannot be filtered out by regression because corresponding reference channels are missed [16]. Furthermore, a regression of the time domain tends to compensate for blinking, for example, which can lead to new artifacts
This approach fails if the measured brain activity has an amplitude comparable to that of the signals caused by blinking.

For this reason, the linear decomposition approach of Bell and Sejnowski is used to correct EEG data. Independent components within a data record can be selected using their Independent Component Analysis (ICA). There are three requirements for performing an ICA: (i) the mixed medium is linear and propagation delays are negligible (ii) the time courses of the sources are independent of each other (iii) the number of sources is equal to the number of their sensors [17]. All these three requirements are given by electroencephalographic data. Thus, the data are linear (i), interfering signals such as heartbeat are not coupled to the sources of EEG activity and thus independent and condition (iii) could be confirmed by numerical simulation [17]. For the analysis of EEG data, the lines of the Input Matrix $\mathbf{x}$ correspond to the signals of the electrodes, the lines of the Output Matrix $\mathbf{u}=\mathbf{W} \mathbf{x}$ correspond to the time curves of the ICA components and the columns of the inverse matrix $\mathbf{W}^{-1}$ indicate the projection force of the respective electrode component. The topography of the signals on the scalp provides information about their formation. Thus, blinking can be found in the front scalp area. In contrast to the PCA, the component time courses of activation are not 
orthogonal. The corrected EEG data are corresponding to $\mathbf{x}^{\prime}=(\mathbf{W})^{-1} \mathbf{u}^{\prime}$, where $\mathbf{u}^{\prime}$ is the matrix of excitation waves, the series with noise signals are set to zero.

\section{Machine Learning Method}

This part of the paper covers spectral analysis and feature extraction, classification and validation. As explained above, the aim of this work was to extract the most predictive frequency bands for the separation of alcoholics and non-alcoholics from existing EEG data.

\section{a) Spectral Analysis and Feature Extraction}

The classical division of the frequency bands into alpha, beta, theta, delta and gamma bands was deliberately not used as a feature extraction criterion in this work. Instead, the frequency range from $0.5 \mathrm{~Hz}$ to $50 \mathrm{~Hz}$ was divided into 99 frequency bands. These each had a span of $0.5 \mathrm{~Hz}$. The hypothesis behind this division is that the information content of finer frequency bands is higher for our concern. Looking at the beta frequency band, it contains a lot of information within the $17.5 \mathrm{~Hz}$ range that is not useful for classifying alcoholics and non-alcoholics. This reduces the relevant information density. However, in order to achieve the best possible classification, the information density must be as high as possible.

The EEG signal cleaned by the ICA must be transformed into a frequency signal [18], which is achieved by the EEG spectral analysis. Here, the EEG signal is reproduced as a function of frequencies with the aid of the Fourier transformation. The EEG signal is first broken down into many sinusoidal oscillations with a known wavelength. Now it is possible to check each wavelength for correspondence with the EEG signal with the aid of correlation analyses. The result of the Fourier transformation is the power spectrum, which allows the distribution of the frequencies of the EEG signal to be estimated [19-20]. Fig 3 describes the complete procedure in simplified form:

a) EEG signal in its original form as a function of time

b) EEG signal is divided into different sinusoidal signals depending on time and displayed between two of the classical frequency bands: delta and theta. Unless the strict division of frequency bands is abandoned, such things go unnoticed. c) Finally the Power spectrum. Now the frequency components of the EEG signal can be recognized. In this example, the highest activity is in the range of $10-12 \mathrm{~Hz}$, which corresponds to the alpha frequency band in Fig. 1.
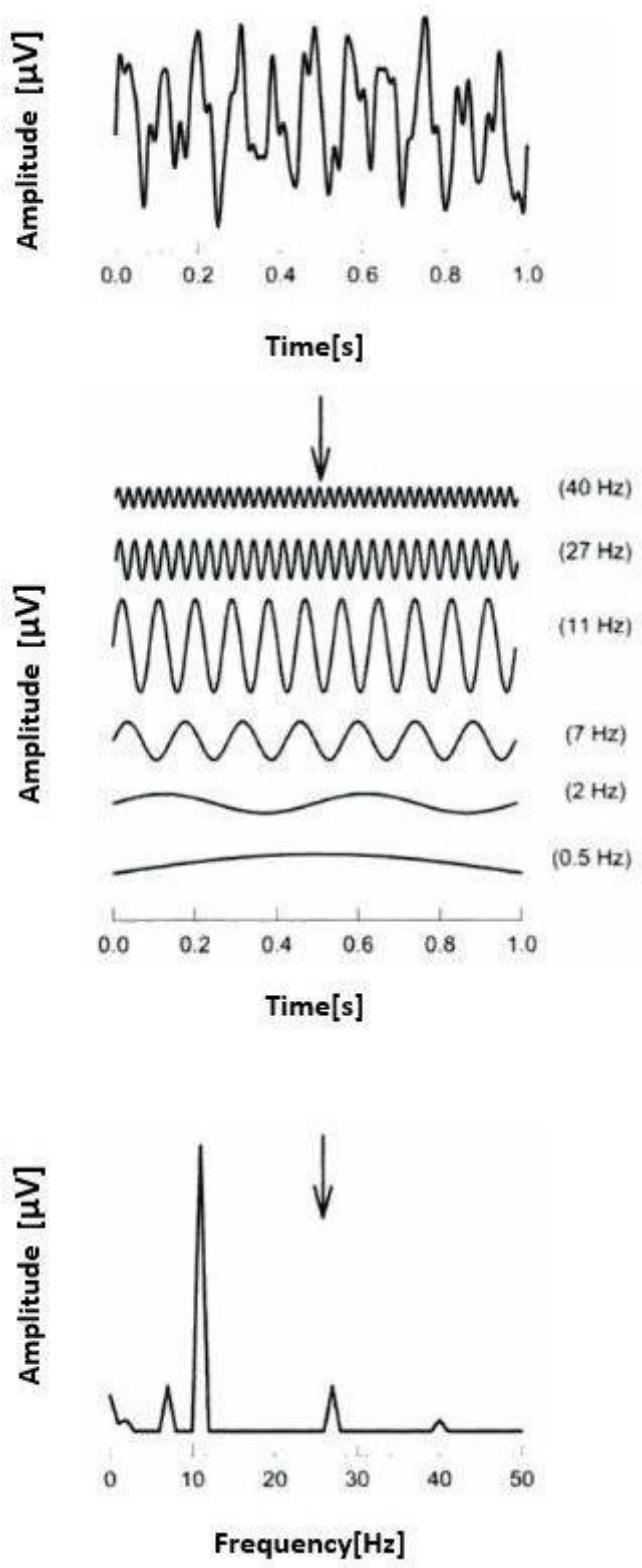

Fig. 3 EEG Signal transformation [19] 


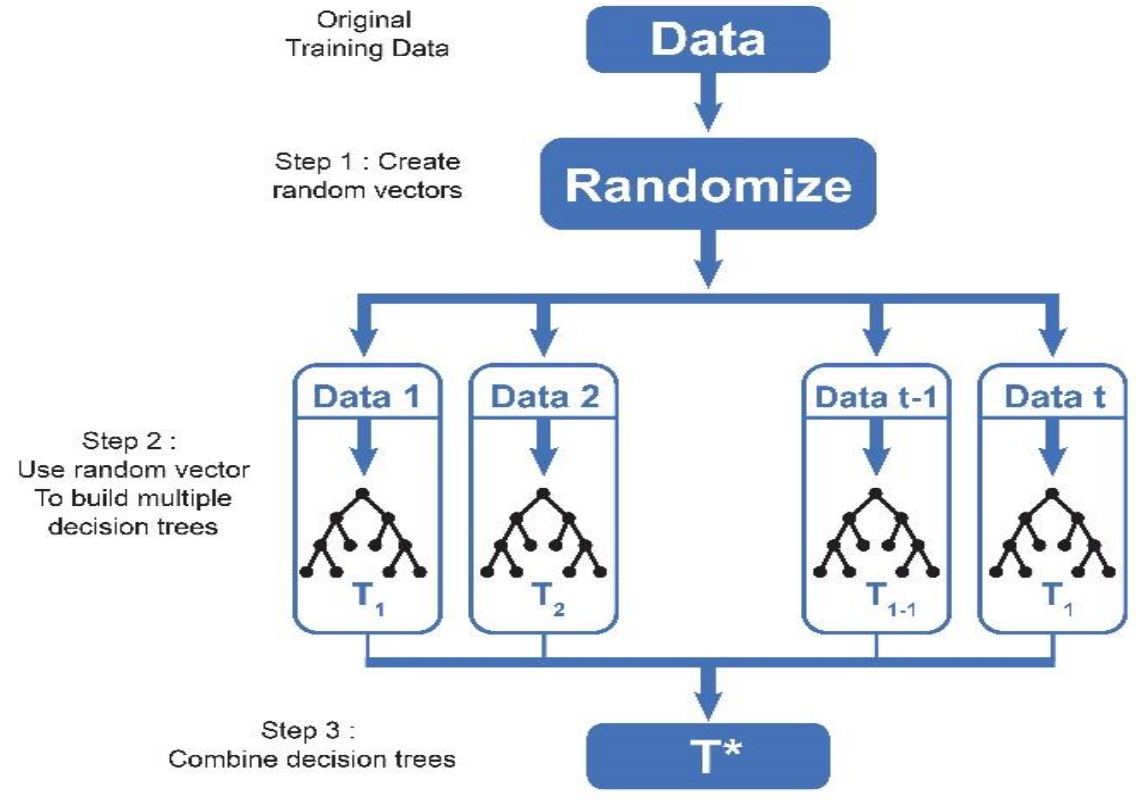

Fig. 4 Random Forest Method

\section{b) Classification}

To answer the question, which are the most important frequency bands for the classification of alcoholism, the Random Forest was chosen as a classifier. Random Forest has made it possible to process the large amount of data efficiently. In addition, it was possible to draw direct conclusions about the most important variables for classification. The Random Forest was originally designed by Breiman [21]. It consists of many individual classification trees, whose individual classification outputs are used to determine the overall classification. The Random Forest process was described by Liaw and Wiener as follows [22]:

1. Draw $n_{\text {tree }}$ bootstrap samples for the original data.

2. For each of the bootstrap samples, an unpruned classification or regression tree grows, with the following modification: at each node, rather than choosing the best split among all predictors, randomly sample $m_{\text {try }}$ of the predictors and choose the best split from among those variables. (Bagging can be thought of as the special case of random forest obtained when $m_{\text {try }}=\mathrm{p}$, the number of predictors)

3. Predict new data by aggregating the predictions of the $n_{\text {tree }}$ trees (i.e., majority votes for classification, average for regression).

The Random Forest of the caret package was used for our classification. The data set was divided as follows:
Training 75\% (92 participants) and test 25\% (30 participants) with 10 repetitions each. The number of trees was $\mathrm{n}=100$ and the variable importance calculated by Random Forest were also displayed. The variable importance represents the statistical significance of each variable in the data related to the influence on the model.

\section{c) Validation}

In order to reach a reliable value from our Random Forest results, the method was supplemented by crossvalidation. Here we worked with the 10 times $10-\mathrm{CV}$. As a result, we were told which subjects were correctly and which incorrectly classified on the basis of the trained model. For this purpose, the cross-validation matrix of the model was generated. The crossvalidation classifies as follows: (i) True positive: The subject is alcoholic and the test has correctly indicated it (ii) False negative: The subject is alcoholic but the model has falsely classified him as non-alcoholic (iii) False positive: The patient is not an alcoholic but the model has classified him as an alcoholic (iv) True negative: The patient is not an alcoholic and has not been classified as an alcoholic by the model either. The cross validation provides information about the robustness of a model. In principle, the 1 truly positive and 4 truly negative values must be highly relative to the total number of test results. Only then can it be guaranteed that the model delivers good quality results. The following key figure is the cross-validation matrix. In addition, the accuracy of the model is calculated. 


\section{Results}

The Random Forest was trained on the data of 92 participants and tested on the data of 30 participants with respective EEG power bands of spans $0.5 \mathrm{~Hz}$. The $10-\mathrm{CV}$ was repeated 10 times on the training set. The result is shown in Table 2 . The trained model with ntry $=100$ and mtry $=50$ has a total accuracy of 96.67 percent (balanced accuracy of 97.4 percent). Prevalence of alcoholics is 36.667 percent.

\begin{tabular}{|c|c|c|c|}
\cline { 3 - 4 } \multicolumn{2}{c|}{} & \multicolumn{2}{c|}{ Reference } \\
\cline { 3 - 4 } \multicolumn{2}{c|}{} & $\begin{array}{c}\text { Non- } \\
\text { alcoholic }\end{array}$ & Alcoholic \\
\hline \multirow{3}{*}{ Predicted } & $\begin{array}{c}\text { Non- } \\
\text { alcoholic }\end{array}$ & 11 & 1 \\
\cline { 2 - 4 } & Alcoholic & 0 & 18 \\
\hline
\end{tabular}

Tab. 2 Confusion Matrix

Our classifier performs very well (Table 3).

\begin{tabular}{lr} 
Performance indicator & Value \\
\hline Accuracy & $96.667 \%$ \\
True positive rate & $94.736 \%$ \\
True negative rate & $100 \%$ \\
Positive predictive value & $91.667 \%$ \\
Negative predictive value & $100 \%$ \\
Balanced accuracy & $97.377 \%$ \\
Kappa & 0.930
\end{tabular}

Tab. 3 Performance of our method

The variable importance is shown in Fig. 5. This has been scaled to 100 . Accordingly, variables 2 to 10 are subtracted relative to the first variable. The most important frequency band is $3.5-4 \mathrm{~Hz}$. Measured by this, the band from $9.5-10 \mathrm{~Hz}$ has a value of 91.77 , the band from 17-17.5 to 90.82. In addition, the following results were achieved: $2.5-3 \mathrm{~Hz} 83.20,11.5-12 \mathrm{~Hz}$ 80.87, 3-3.5 Hz 80.29. The frequency bands $6.5-7 \mathrm{~Hz}$, 4.5-5 Hz, $9-9.5 \mathrm{~Hz}$ and $1.5-2 \mathrm{~Hz}$ have a relative variable importance of 72.39 to 79.82 . Overall 74 frequency bands were below a relative variable importance of 50. Looking at the classical frequency bands, the most important variables are as follows: three variables in the theta range, three variables in the alpha range, three variables in the delta range and one variable in the beta range [Fig. 6].

\section{Discussion}

This paper proposes a method with a balanced accuracy of 97.4 percent to identify alcoholics from
EEG data. Unlike in the other cited papers, the classical frequency bands with the highest accuracy are not given, but go much deeper into the specific frequency ranges. Looking at the literature to date on the same topic, there is often no agreement as to which of the classical frequency bands is most relevant for the diagnosis of alcoholism. Ehlers and Phillips[23] see the alphaband as extremely relevant. Here a lower spectral power would be a signal for alcoholism. This research is also taken up by Bernice Projesz et al. [24]. However, he also regards the thetaband as extremely predictive. Here, strong drinkers would have more synchronization with their eyes closed than nonalcoholics. Madhavi Rangaswamy et al. [25] also see a great deal of predictive power in the thetaband. Bernice Projesz et al. [24] explain that despite the good results with the thetaband, the beta frequency has become a strong indicator of alcoholism among scientists and medical professionals. However, the results of Wajid Mumtaz et al. [10] see a good classifier of alcoholics and non-alcoholics in the thetaband and the Hi-Gamma bands $(30-40 \mathrm{~Hz})$. Eveline A. de Bruin et al. [26] analyze the EEG data of heavy drinking students compared to light drinking students and also comes to the conclusion that the EEG data of heavy drinking students, especially in theta and Gamma band, differ enormously from the EEG data of the control group.

The hypothesis of this paper was that a more detailed distribution of frequencies increases the quality of the available information and thus enables a better classification of alcoholics and non-alcoholics. The literature mentioned here only names the classical frequency bands as alcoholism indicators. As explained, there is disagreement over the best frequency band for use as an indicator. With our new approach we wanted to show that highly informative frequency ranges are available within the entire spectrum, independent of the classical frequency bands. Looking at the accuracy of the Random Forest, this finer frequency distribution proves us to be right: a classification based on our model is correct with a probability of $97.4 \%$. Far more interesting, however, is where the 10 most important classifiers are found in the spectral range. Thus, three important variables can be found in the delta area, which has been almost ignored in previous publications. Looking at the information generated by an EEG, it seems fatal to ignore the delta area in classifications, since a large part of the data is generated in this area. The fact that the delta area has not played a role in the previous classification of alcoholics and non-alcoholics may have to do with the hypothesis mentioned above. With a large amount of data generated in the delta area, the density of relevant classification information 
decreases. This problem can be resolved using our smaller division. Looking at the other results, there are also three variables in the alpha range. In the work of Wajid Mumtaz et al., the alpha range achieved an accuracy of 75.4 with linear regression of the alcoholics and control group. very important frequency ranges were found, mainly in the Delta, Theta, Alpha and Beta I+II spectra.

While previous work on the same dataset achieved accuracies between 78 and 95.83 percent (see Table 4), our approach significantly outperforms with 97.4 percent (balanced accuracy).

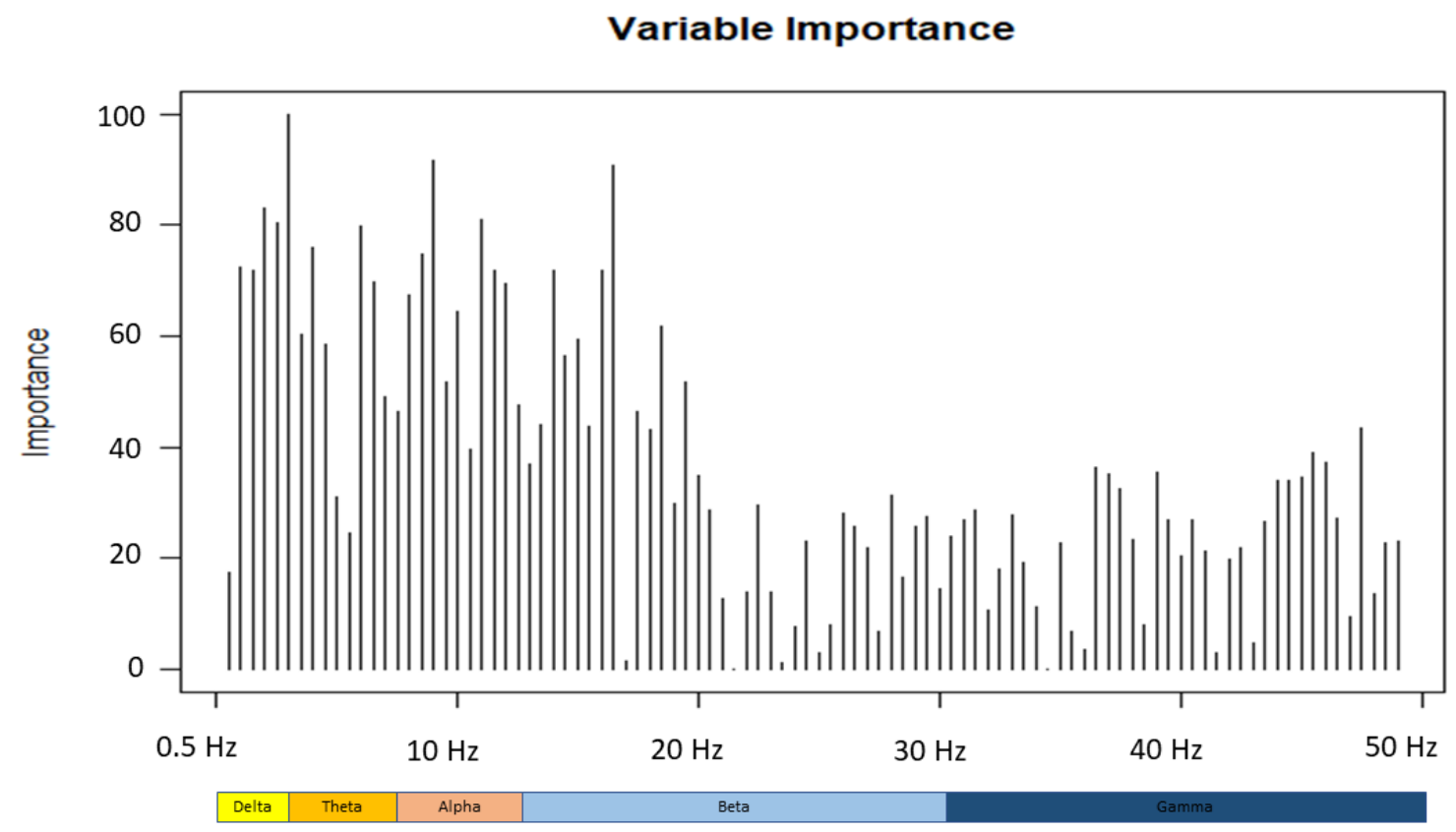

Fig. 5 Variable Importance

\begin{tabular}{|c|c|c|c|c|c|c|c|c|c|c|c|c|c|c|c|c|c|c|}
\hline Importance & & & & & & & & & & & & & & & & & & \\
\hline $\mathrm{Hz}$ & 1 & 2 & 3 & 4 & 5 & 6 & 7 & 8 & 9 & 10 & 11 & 12 & 13 & 14 & 15 & 16 & 17 & 18 \\
\hline Band & \multicolumn{3}{|c|}{ Delta } & \multicolumn{4}{|c|}{ Theta } & \multicolumn{5}{|c|}{ Alpha } & \multicolumn{6}{|c|}{ Beta } \\
\hline
\end{tabular}

\begin{tabular}{|c|c|c|c|c|c|c|c|c|c|c|c|c|c|c|c|c|c|c|}
\hline & & & & & & & & & & & & & & & & & & \\
\hline 26 & 27 & 28 & 29 & 30 & 31 & 32 & 33 & 34 & 35 & 36 & 37 & 38 & 39 & 40 & 41 & 42 & 43 & $\frac{1}{44}$ \\
\hline & & eta & & & & & & & & & Gar & & & & & & & \\
\hline
\end{tabular}

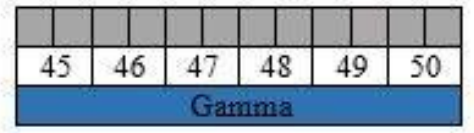

Fig. 6 Top most predictive bandwidths marked in green

While the thetaband seems to be of great relevance to us with three variables, only one variable can be found in the beta area. Overall, the results show why it may be appropriate to depart from the classical medical distribution of the frequency bands of the EEG. Another reason for this is the strict transitions of the frequency bands. Looking at our results, a cluster of

\begin{tabular}{|c|l|l|l|}
\hline Year & Reference & Method & Accuracy \\
\hline 2007 & $\begin{array}{l}\text { Yazdani \& } \\
\text { Setarehdan } \\
{[27]}\end{array}$ & $\begin{array}{l}\text { k-Nearest-Neighbor, } \\
\text { Naive Bayes }\end{array}$ & $87.5 \%$ \\
\hline 2012 & $\begin{array}{l}\text { Acharya } \\
{[28]}\end{array}$ & $\begin{array}{l}\text { Support Vector } \\
\text { Machine }\end{array}$ & $91.7 \%$ \\
\hline 2015 & $\begin{array}{l}\text { Gopan et } \\
\text { al. [29] }\end{array}$ & $\begin{array}{l}\text { Support Vector } \\
\text { Machine, Fuzzy k- } \\
\text { Nearest-Neighbor }\end{array}$ & $\begin{array}{l}\text { SVM: 78-95\%, } \\
\text { Fuzzy } \\
\text { k-NN: 77-88\% }\end{array}$ \\
\hline 2017 & $\begin{array}{l}\text { Bajaj et al. } \\
{[30]}\end{array}$ & $\begin{array}{l}\text { Non-Negative Least } \\
\text { Squares }\end{array}$ & 95.83\% \\
\hline
\end{tabular}

Tab. 4 Related work on the same dataset 


\section{Conclusion and Outlook}

In the context of this work it should be shown that ML methods for the classification of alcoholics and nonalcoholics can achieve even better results using EEG features if the classical frequency bands are divided into smaller frequency bands. The results of this work have confirmed the hypothesis that the information content increases significantly as a result of this division. The results of this work are to be improved in the future by even more detailed observations of the smallest frequencies of the EEG electrodes. In addition, the individual electrodes will play a more important role in further work. These can be used to identify the predictive brain areas and thus to better predict long-term brain damage caused by alcoholism. Furthermore, the results of this work can be used in the search for endangered patients for diagnosis. Today, EEG diagnoses are mainly used in nine large fields: Diagnosis of epilepsy, dementia, brain tumors, strokes, autism, insomnia, alcoholism, anesthesia and coma and brain death [31]. All these nine fields have a very high impact on patients' lives. For this reason it is of the utmost importance to diagnose with extreme care. Technical assistance could help doctors to make faster, more detailed decisions. On the one hand, this provides relief for doctors, who can make their decisions on the basis of more valid key figures. On the other hand, it reduces the likelihood of human error in his medical field and thus helps the respective patient. The field of research can therefore also be extended to other parts of EEG diagnosis. Dementia will be the main focus of further work and can build on existing work [32].

In addition, in future work we will transfer this novel pre-processing step (unfolding outdated standard bandwidths in fine-graded spectrums) prior to entering a Random Forests classifier to other applications outside EEG, such as pupillary hippus for user performance and cognitive load assessment [33-35], and frequencies of facial actions for cognitive load evaluation [36].

Furthermore, in future work we will transfer the novel pre-processing step (fine-graded spectrums) to feed other machine learning classifiers.

\section{References}

[1] C. Lange, K. Manz, B. Kunt, "Alcohol consumption among adults in Germany: risky drinking levels", Journal of Health Monitoring, no. 2, pp. 64-69, 2017.
[2] NIH National Institute on Alcohol Abuse and Alcoholism, "Beyond Hangovers - understanding Alcohol's impact on your health", NIH Publication, no. 15, pp .6-8, 2010.

[3] S. Siuly, Li Yan, "Designing a robust feature extraction method based on optimum allocation and principal component analysis for epileptic EEG signal classification", Computer Methods Program in Biomedicine, vol. 119, no.1, pp. 29-42, 2015.

[4] S. Siuly, Li Yan, "A novel statistical framework for multiclass EEG signal classification", Engineering Applications of Artificial Intelligence, vol. 34, pp. 154-167, 2014.

[5] C. Ehlers, T. Reed, "Ethanol effects on EEG spectra in monkeys: comparison to morphine and diazepam", Electroencephalography Clinical Neurophysiology, vol. 66, no.3, pp. 317-321, 1987.

[6] M. Teplan, "Fundamentals of EEG Measurement", IEEE Measurement Science Review, vol. 2, pp. 1-11, 2002.

[7] Freeman W. J, Quiroga Q.R., Imaging Brain Function with EEG: Advanced Temporal and Spatial Analysis of Electroencephalographic Signals, New York, NY, USA: Springer Science \& Business Media, 2012.

[8] Harung H. S, Travis F., Excellence through Mind-Brain Development: The Secrets of World-Class Performers, London, England: Taylor \& Francis Ltd., 2016.

[9] Sun Y., Ning Y., Xinhe X, "EEG analysis of alcoholics and controls based on feature extraction", in 8th international Conference on Signal Processing, 2007.

[10] Mumtaz W., Vuong P., Xia L., Malik A., Rashid R., "An EEG-based machine learing method to screen alcohol use disorder", Cognitive neurodynamics, vol. 11, no. 2, pp. $161-171$

2017.

[11] G. Gopika, S. Neelam, Babu D. J, "EEG signal classification in non-linear framework with filtered training data" in $23^{\text {rd }}$ European Signal Processing Conference, 2015, pp. 624-628.

[12] X. L. Zhang, H. Begleiter, B. Porjesz, W. Wang, A. Litke, "Event Related Potentials During Object Recognition Tasks", Brain Research Bulletin, vol. 38, no. 6, pp. 531-538, 1995.

[13] C. D. Binnie, A. J. Rowan, T. H. Gutter, A Manual of Electroencephalographic Technology, Cambridge: Cambridge University Press, 1982.

[14] G. Deuschl, A. Eisen, Recommendations for the practice of clinical neurophysiology: guidelines of the International Federation of Clinical Neurophysiology, Amsterdam: Elsevier, 1999. 
[15] S. P. Fitzgibbon, D. M. W. Powers, K. J. Pope, C. R. Clark, "Removal of EEG Noise and Artifact Using Blind Source Separation", Journal of Clinical Neurophysiology, vol. 24, no. 3, pp. 232-243, 2007.

[16] Jung T.P., Humphries C., Lee T., Makeig S., Mckeown M., Iragui V., Sejnowski T., "Extended ICA Removes Artifacts from Electroencephalographic Recordings", Advances in Neural Information Processing Systems, vol. 10, pp. 894-900, 1997.

[17] T. Nakada, Integrated Human Brain Science: Theory, Method, Applications, Tech. Rep. INC-9606, Institute for Neural Computation, San Diego, CA, 1996.

[18] A. Delorme, S. Makeig, "EEGLAB: an open source toolbox for analysis of single-trial EEG dynamics including independent component analysis", Journal of Neuroscience Methods, vol. 134, no. 1, pp. 9-24, 2004.

[19] van Vugt M., Sederberg P., Kahana M., "Comparison of spectral analysis methods for characterizing brain oscillations", Journal of Neuroscience Methods, vol. 162, no. 1-2, pp. 49-63, 2007.

[20] M. Ahirwal, N. Londhe., "Power Spectrum Analysis of EEG Signals for Estimating Visual Attention", International Journal of Computer Applications, vol. 42, no.15, pp. 22-25, 2012.

[21] L. Breiman, "Random forests", Machine Learning, Machine Learning, vol. 45, no. 1, pp. 5-32, 2001.

[22] A. Liaw, M. Wiener, "Classification and Regression by random Forest”, R News 2, Vol. 2/3, pp. 18-22, 2002.

[23] L. Ehlers, Cindy \& Phillips, Evelyn, "Association of EEG alpha variants and alpha power with alcohol dependence in Mexican American Young Adults", Alcohol (Fayetteville, N.Y.), Vol. 41 (1), pp. 13-20, 2007.

[24] B. Porjesz, M.Rangaswamy, C. Kamarajan, K. A. Jones, A. Padmanabhapillai, H. Begleiter, "The utility of neurophysiological markers in the study of alcoholism", Clinical Neurophysiology, vol. 116, pp. 993-1018, 2005.

[25] M. Rangaswamy, B. Porjesz, D. Chorlian, K. Choi, K. Jones, K. Wang, J. Rohrbaugh, S. O'Connor, S. Kuperman , T. Reich, H. Begleiter, "Theta Power in the EEG of Alcoholics", ALCOHOLISM: Clinical and experimental research, vol. 27, no. 4, pp. 607-615, 2003.

[26] E. de Bruin, S. Bijl, C. Stam, K. Böcker, L. Kenemans, M. Verbaten, "Abnormal EEG synchronisation in heavily drinking students", Clinical Neurophysiology, vol. 115, pp. 2048-2055, 2004.

[27] A. Yazdani, S. K. Setarehdan, "Classification of EEG signals correlated with alcohol abusers", Proc. of 9th International Symposium on Signal Processing and Its Applications, Sharjah, pp. 1-4, 2007.

[28] U. R. Acharya, S. V. Sree, S. Chattopadhyay, J. S. Suri, "Automated diagnosis of normal and alcoholic EEG signals", International Journal of Neural Systems, vol. 22, no. 3, 1250011

[29] K. G. Gopan, N. Sinha, J. D. Babu, "EEG signal classification in non-linear framework with filtered training data", In 23rd European Signal Processing Conference (EUSIPCO), Nice, pp. 624-628, 2015.

[30] V. Bajaj, Y. Guo, A. Sengur, S. Siuly, O. F. Alcin, “A hybrid method based on time-frequency images for classification of alcohol and control EEG signals", Neural Computing and Applications, vol. 28, no. 12, pp. 3717-3723, 2017

[31] S. Siuly, Y. Li, Y. Zhang, "EEG Signal Analysis and Classification: Techniques and Applications", Springer International Publishing, 2016.

[32] K. Tsoi, J. Chan, H. Hirai, S. Wong, T. Kwok, "Cognitive tests to detect dementia: A systematic review and meta-analysis", JAMA Internal Medicine, vol. 175, no. 9, pp. 1450-1458, 2015.

[33] R. Buettner, S. Sauer, C. Maier, A. Eckhardt, "Real-time Prediction of User Performance based on Pupillary Assessment via Eye-Trackings", AIS Transactions on Human-Computer Interaction, vol. 10, no. 1, pp. 26-56, 2018.

[34] R. Buettner, "Analyzing Mental Workload States on the Basis of the Pupillary Hippus", In NeuroIS Proceedings: Gmunden Retreat on NeuroIS 2014, June 5-7, 2014, Gmunden, Austria, p. 52, 2014.

[35] R. Buettner, "Asking both the User's Brain and its Owner using Subjective and Objective Psychophysiological NeuroIS Instruments", ICIS 2017 Proceedings: 38th International Conference on Information Systems, 2017.

[36] R. Buettner, "Robust user identification based on facial action units unaffected by users' emotions", HICSS-51 Proceedings: 51th Hawaii International Conference on System Sciences, pp. 265-273, 2018. 